\title{
Formation mechanism of a degenerate thin layer at the interface of a GaN/sapphire system
}

\author{
X. L. Xu, ${ }^{\text {a) }}$ C. D. Beling, S. Fung, ${ }^{\text {b) }}$ and Y. W. Zhao \\ Department of Physics, the University of Hong Kong, Hong Kong, People's Republic of China \\ N. F. Sun, T. N. Sun, and Q. L. Zhang \\ Hebei Semiconductor Institute, Shijiazhuang, Hebei, People's Republic of China
}

H. H. Zhan, B. Q. Sun, J. N. Wang, and W. K. Ge

Department of Physics, Hong Kong University of Science and Technology, Hong Kong, People's Republic of China

P. C. Wong

MCPF, Hong Kong University of Science and Technology, Hong Kong, People's Republic of China

(Received 4 May 1999; accepted for publication 10 November 1999)

\begin{abstract}
It has recently been suggested that the thin degenerate layer found at the GaN/sapphire interface results from a high concentration of stacking faults. The studies of this letter, however, show that this is not the most likely explanation for the presence of such a degenerate layer. Using x-ray energy-dispersive spectroscopy and secondary ion-mass spectroscopy, profile distributions of elements $\mathrm{Ga}, \mathrm{N}, \mathrm{O}, \mathrm{C}$, and $\mathrm{Al}$, near the interface, have been obtained. The distributions reveal very high $\mathrm{O}$ and $\mathrm{Al}$ concentrations in the GaN film within $0.2 \mu \mathrm{m}$ from the interface, together with a material depletion of Ga and N. Such conditions strongly favor $n^{+}$conductivity in this interfacial region because not only are $\mathrm{N}$-vacancy and $\mathrm{N}$-site $\mathrm{O}$ donors present, but $\mathrm{Al}$ incorporated on the $\mathrm{Ga}$ sublattice reduces the concentration of compensating Ga-vacancy acceptors. The two-layer (film plus interface) conduction has been modeled, and the effect of conduction in the GaN film thus isolated. (C) 2000 American Institute of Physics. [S0003-6951(00)00302-8]
\end{abstract}

Most of the GaN (also InGaN and AlGaN) material used so far for device development consists of epitaxial growth on sapphire. Because of the large lattice mismatch (14\%) between $\mathrm{GaN}$ and sapphire, a thin, highly dislocated region is generated at the layer/substrate interface to relieve the strain, and the structural properties of this interface region have been studied in great detail. ${ }^{1}$ However, little is known at present about the electrical and optical properties of the interface.

Gotz et $a .^{2}{ }^{2}$ have progressively thinned a $13-\mu \mathrm{m}$-thick HVPE layer down to $1.2 \mu \mathrm{m}$, and their results have suggested that a significant high donor concentration must exist near the interface. By performing Hall measurements, as the layer thickness in their sample was reduced from 13 to 1.2 $\mu \mathrm{m}$, these authors using a two-layer model ${ }^{3}$ estimated that the sheet concentration of donors in the interfacial region exceeded $10^{15} \mathrm{~cm}^{-2}$. Capacitance-voltage measurements, on the other hand, showed that $\mathrm{N}_{D}-\mathrm{N}_{A}$ for the 13- $\mu \mathrm{m}$-thick bulk film was relatively constant at about $5 \times 10^{16} \mathrm{~cm}^{-3}$, equally with a much lower sheet concentration of only about $7 \times 10^{13} \mathrm{~cm}^{-2}$. The conclusion was, therefore, that the Hall measurements were being strongly affected by an interfacial region.

In this letter, the formation mechanism of the interfacial $n^{+}$layer will be discussed; a subject not yet considered in

\footnotetext{
a) Also at the Department of Physics, the University of Science and Technology of China, Hefei, Anhui, P.R.C.

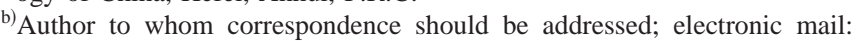
sfung@hkucc.hku.hk
}

the published literature. The discussion focuses on profile distributions of elements $\mathrm{Ga}, \mathrm{N}, \mathrm{O}, \mathrm{Al}$, etc., near the interfacial region as obtained by $\mathrm{x}$-ray energy-dispersive spectroscopy (XEDS) and secondary ion-mass spectroscopy (SIMS). These data suggest extremely high concentrations of $\mathrm{Ga}$ and $\mathrm{N}$ vacancies close to the interface in the $\mathrm{GaN}$ film. It is argued that the $\mathrm{N}$-vacancy together with $\mathrm{N}$-site $\mathrm{O}$ form dominant donors and sources of electrons. It is further argued that while the Ga vacancy would tend to compensate the donors, the compensation ratio could be greatly reduced with Al diffusing from the sapphire, incorporating at the Ga sites. Finally, the method used previously ${ }^{4}$ for fitting both mobility and carrier concentration data will be presented but within the framework of the two-layer model. The agreement with experimental data is shown to be excellent.

The samples used in the present work were grown by the two-flow MOCVD method, which has been described in a previous work. ${ }^{5}$ In particular, a $300 \AA \mathrm{GaN}$ buffer layer was grown between the GaN film and the sapphire substrate of (0001) orientation. Prior to growing the buffer layer, the substrate was hydrogenated at $1050{ }^{\circ} \mathrm{C}$. The thickness of the $\mathrm{GaN}$ film was $2.0 \mu \mathrm{m}$. The Hall measurements are the same as those in Ref. 4. The XEDS measurements were carried out by a scanning electron microbeam (SEM) (Cambridge Co.). An electron beam of $100 \AA$ diam was scanned across the sample's lateral cross section in order to find the atomic concentrations across the interface. The appropriate $Z A F$ [atomic number $(Z)$, absorption $(A)$, and fluorescence $(F)]$ correction factors were made to the XEDS data to obtain the correct atomic concentrations. ${ }^{5}$ 


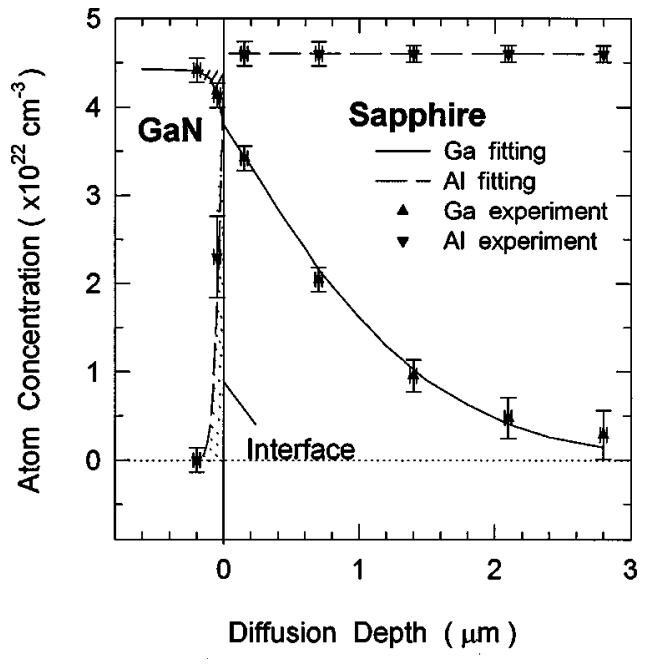

FIG. 1. Concentration distributions of $\mathrm{Ga}$ and $\mathrm{Al}$ as a function of depth obtained from XEDS. The Ga diffusion into sapphire and $\mathrm{Al}$ diffusion into $\mathrm{GaN}$ are fitted by complementary error functions. The Ga vacancies and the back-diffused $\mathrm{Al}$ are expressed by the oblique real lines and the oblique dashed lines, respectively.

Figure 1 shows the detail of the $\mathrm{Ga}$ and $\mathrm{Al}$ atomic profiles as obtained from XEDS spectra near the interface of the GaN-sapphire system, where $x<0, x=0$, and $x>0$ correspond to the GaN film, the interface, and the sapphire substrate, respectively. The absolute concentration scales of Fig. 1 are obtained assuming a negligible concentration of vacancies in the film and substrate, an $\mathrm{Al}$ concentration of 4.6 $\times 10^{22} \mathrm{~cm}^{-3}$ in the sapphire $\left(\mathrm{Al}_{2} \mathrm{O}_{3}\right)$, and a $\mathrm{Ga}$ concentration of $4.5 \times 10^{22} \mathrm{~cm}^{-3}$ in the GaN film. As in our previous work, ${ }^{5}$ a large amount of Ga diffusion into the sapphire is seen to have occurred with a falloff distance $\sim 1 \mu \mathrm{m}$. It is also seen that the $\mathrm{Ga}$ concentration decreases from its bulk value of $4.5 \times 10^{22} \mathrm{~cm}^{-3}$ at $x=-0.2 \mu \mathrm{m}$ to $3.7 \times 10^{22} \mathrm{~cm}^{-3}$ at $x=0$, indicating an appreciable Ga deficiency in this range. This deficiency on its own would not account for the quantity of $\mathrm{Ga}$ in the sapphire and it appears that $\mathrm{Ga}$ is diffusing out of the bulk film. The large Ga concentration gradient on the film side of the interface confirms this view. In the ideal case of the Ga deficiency being in the form of $V_{\mathrm{Ga}}$ we obtain $\left[V_{\mathrm{ga}}\right]=2.2 \times 10^{21} \mathrm{~cm}^{-3}$, and for the average concentration of $\mathrm{Al}$ in the interface region a value of $1.4 \times 10^{22} \mathrm{~cm}^{-3}$ which is over six times greater than $\left[V_{\mathrm{Ga}}\right]$. This concentration ratio suggests that the Ga vacancies [often taken to be the main source of acceptors in undoped GaN (Ref. 6)] could be totally replaced by the diffused $\mathrm{Al}$, even though only one sixth of the $\mathrm{Al}$ can substitute for the same group-III Ga atoms. It is noted that for the epitaxy methods, such as MOCVD and HVPE, rather high temperatures (higher than $800^{\circ} \mathrm{C}$ ) are required for growth. At such sustained elevated temperatures $\left(T=1050^{\circ} \mathrm{C}\right.$ for MOCVD), atomic diffusion would appear to be a dominant process associated with the film's growth allowing $\mathrm{Al}$ atoms to diffuse to and fill the $\mathrm{Ga}$ sites in the near-interface region in the film.

In order to obtain a more detailed picture of the defects or impurities present in the $n$-GaN film, we investigated a depth profile of five elements $(\mathrm{Ga}, \mathrm{N}, \mathrm{O}, \mathrm{Al}$, and $\mathrm{C})$ using SIMS. As shown in Fig. 2, the intensities of the five elements are only relative because of their different SIMS yields. However, we can obtain absolute concentration distribution Downloaded 02 Nov 2006 to 147.8.21.97. Redistribution subject

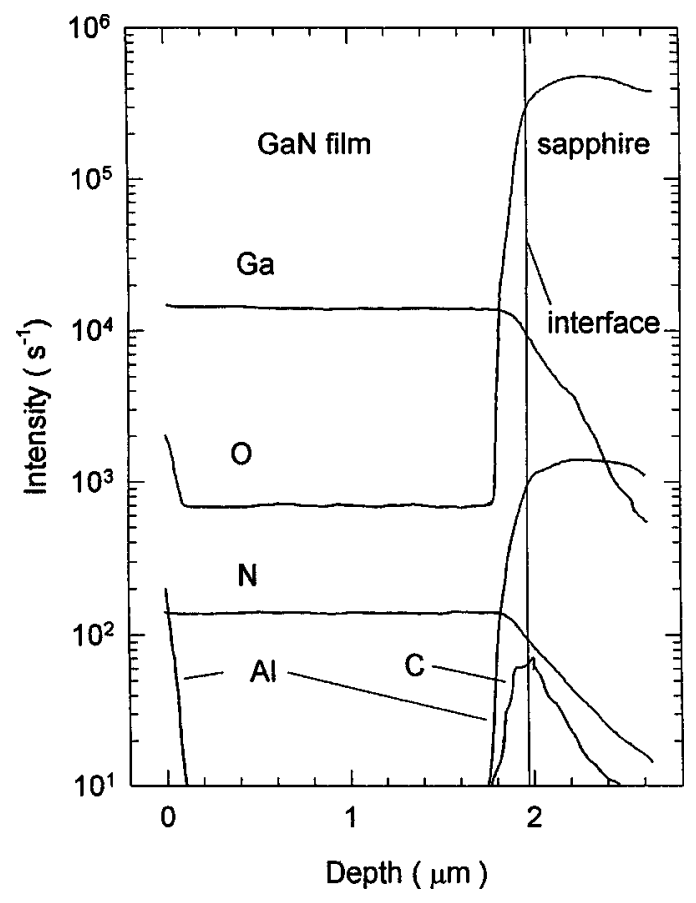

FIG. 2. SIMS depth profile of an $n$-GaN film on a sapphire substrate. The intensities of the five elements are only relative because of their different yields in SIMS. The high intensities of $\mathrm{O}$ and $\mathrm{Al}$ near the surface are due to a segregation effect.

of $\mathrm{Ga}, \mathrm{N}, \mathrm{Al}$, and $\mathrm{O}$ based on the following approximations:

(i) The concentrations of $\mathrm{Ga}$ vacancies and $\mathrm{N}$ vacancies $\left(V_{\mathrm{Ga}}\right.$ and $V_{\mathrm{N}}$ ) in the $\mathrm{GaN}$ film, which are at some distance from the interface region $(\sim 0.2 \mu \mathrm{m}$, from the SIMS spectra), are not high (i.e., they are less than $10^{22} \mathrm{~cm}^{-3}$ ). Based on this reasonable assumption, the concentration of $\mathrm{Ga}($ or $\mathrm{N})$ in the film is $4.5 \times 10^{22} \mathrm{~cm}^{-3}$.

(ii) The diffusing source of $\mathrm{Al}$ and $\mathrm{O}$ is from the sapphire substrate. This is due to the fact that the diffusion is associated with the film growth process. ${ }^{5}$ Absolute concentrations of $\mathrm{Al}$ and $\mathrm{O}$ in the film may, therefore, be obtained by referencing to the known values in the sapphire, namely, $4.6 \times 10^{22}$ and $6.9 \times 10^{22} \mathrm{~cm}^{-3}$, respectively.

Based on these assumptions, the calculated average concentrations of $V_{\mathrm{Ga}}, V_{\mathrm{N}}, \mathrm{O}$, and $\mathrm{Al}$ in the interface region are $5 \times 10^{21} \mathrm{~cm}^{-3}, 5 \times 10^{21} \mathrm{~cm}^{-3}, 2.4 \times 10^{22} \mathrm{~cm}^{-3}$, and 3.5 $\times 10^{22} \mathrm{~cm}^{-3}$, respectively. As with the XEDS data, these concentrations lead to an expectation that most Ga vacancy sites are replaced by the group-III element $\mathrm{Al}$ at the interface. This follows not only from the copious amount of $\mathrm{Al}$ present at the interface, but also from the fact that $\mathrm{Al}$ has similar chemistry to Ga. Since the Ga vacancy is believed to be a dominant acceptor in $\mathrm{GaN}$, it then follows that the compensation ratio of the interface layer would be greatly reduced.

Turning now to donor sites, we see an opposite tendency. If the main source of the donor is from the $\mathrm{N}$ vacancy ${ }^{7}$ or from the $\mathrm{N}$-site oxygen, ${ }^{8}$ the donor concentration could be extremely high in the interface layer with donor sites present at the $10^{21}-10^{22} \mathrm{~cm}^{-3}$ level and with the aforementioned reduction in $V_{\mathrm{Ga}}$ acceptor sites, an extremely high $\left(\sim 10^{21} \mathrm{~cm}^{-3}\right)$ electron density would be expected. Clearly, this electron concentration would be degenerate and temperature independent, since the Mott concentration in AlP license or copyright, see http://apl.aip.org/apl/copyright.jsp 
GaN is about $1 \times 10^{18} \mathrm{~cm}^{-3},{ }^{9}$ and the concentration at which the Fermi level enters the conduction band is about 6 $\times 10^{18} \mathrm{~cm}^{-3}{ }^{10}$

The above arguments explain why, on the basis of atomic interdiffusion during growth, a thin $n^{+}$interface layer forms between the sapphire and the GaN film. The XEDS and SIMS data both suggest the thickness of this layer to be about $0.2 \mu \mathrm{m}$. It is with this thin degenerate layer in mind that attention is now turned to modeling its effect on Hall measurement data.

The temperature-independent mobility $\mu_{2}$ and concentration $n_{2}$ (from the interface layer properties) allow an easy separation of the bulk-film Hall properties, $\mu_{H 1}$ and $n_{H 1}$, from the measured two-layer properties, $\mu_{H}$ and $n_{H} \cdot{ }^{3}$ Using this two layer model, our Hall data give an interfacial carrier density $n_{H 2}=2.2 \times 10^{20} \mathrm{~cm}^{-3}$, which is lower than that obtained from the XEDS and SIMS results of $\sim 10^{21} \mathrm{~cm}^{-3}$. The most likely explanation is that the $\mathrm{GaN}$ of the interface layer is not of perfect crystal structure. The lattice mismatch and different thermal conductivity between the GaN film and sapphire is so large that a "quasicrystalline" layer results. ${ }^{1}$ The carrier concentration is lower than that of $V_{\mathrm{N}}$ and N-site oxygen partly because, in such an imperfect layer, only some of the latter are electrically active and partly because the dislocations present would trap carriers. From Eqs. (1) and (2), the extracted film concentration $n_{H 1}$ and mobility $\mu_{H 1}$ of a typical sample are plotted in Figs. 3(a) and 3(b), respectively.

To fit the carrier concentration data, we use a twodonor model. ${ }^{4}$ Based on photoluminescence (PL) measurements, ${ }^{11,12}$ the second is a deeper shallow donor with $E_{C}-E_{2}=42 \mathrm{meV}$. Figure 3(b) shows the corrected room temperature $\mu_{H 1}$ to be $430 \mathrm{~cm}^{2} \mathrm{~V}^{-1} \mathrm{~s}^{-1}$ in good agreement with the compensation ratio $C=0.6$ (Ref. 13) (for the calculated results of the carrier-concentration-dependent compensation ratio, see Ref. 13 for details). If we choose $\mathrm{N}_{D 1}=3.0$ $\times 10^{17} \mathrm{~cm}^{-3}$ (which corresponds to the value of the $\mu_{H 1}$ ), one obtains $\mathrm{N}_{A}=0.6 \mathrm{~N}_{D 1}=1.8 \times 10^{17} \mathrm{~cm}^{-3}$. Moreover, the first donor level may be obtained as $E_{C}-E_{1}=5 \times 10^{-15}$ $\mathrm{N}_{D 1}^{2 / 3}=1.8 \mathrm{meV}$ (formula from Ref. 14). According to Ilegems and Montgomery, ${ }^{15}$ the saturation temperature for complete donor ionization is $T \sim 1000 \mathrm{~K}$. Extension of the measurement data to this temperature suggests a value of $\mathrm{N}_{D 2}=1.0 \times 10^{18} \mathrm{~cm}^{-3}$.

It is found that the corrected mobility data [Fig. 3(b)] are fitted well by solutions of the Boltzmann equations in the relaxation time approximation assuming ionized impurity (ii), deformation potential (dp), piezoelectric (pe), and polaroptical phonon (po) scattering. ${ }^{2,4}$ The good fit is obtained by choosing the above values of $E_{C}-E_{1}, E_{C}-E 2, \mathrm{~N}_{A}, \mathrm{~N}_{D}$, and $n_{C}$ in the calculation. The scattering constants are taken from Ref. 16, and references therein: acoustic potential $E_{1}$ $=9.2 \mathrm{eV}$, piezoelectric constant $h_{\mathrm{pz}}=0.5 \mathrm{C} / \mathrm{m}^{2}$, dielectric constant $\epsilon_{\infty}=5.47 \epsilon_{0}$ and $\epsilon_{l f}=10.4 \epsilon_{0}$, effective mass $m^{*}$ $=0.22 \mathrm{~m}_{0}$, speed of sound $s=6.59 \times 10^{3} \mathrm{~m} \mathrm{~s}^{-1}$, mass density $\rho=6.10 \times 10^{3} \mathrm{~kg} \mathrm{~m}^{-3}$, and Debye temperature $T_{D}=1044 \mathrm{~K}$.

One of the authors (S. Fung) wishes to acknowledge valuable financial support from the HKU CRCG and the Hong Kong RGC research grants.
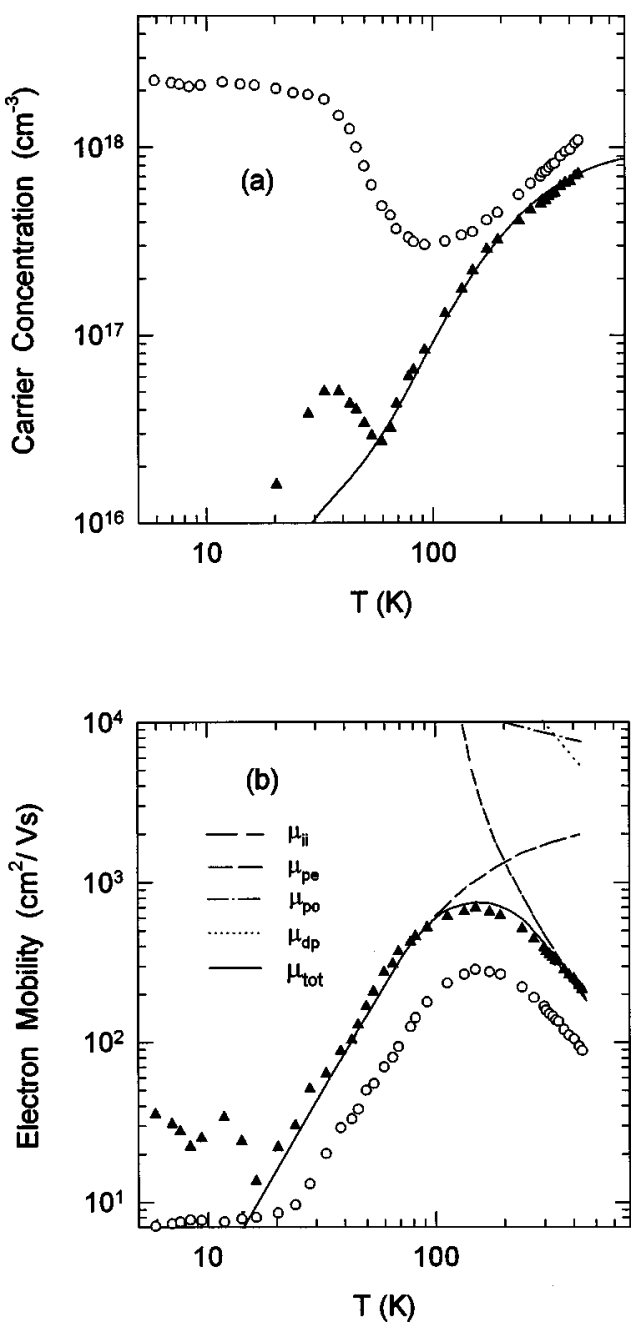

FIG. 3. (a) Temperature dependence of the uncorrected Hall carrier concentration (open circles), and the corrected data (triangles) with the interfacial conduction subtracted. The line shows the model fit to the data as described in the test. (b) Temperature dependence of the uncorrected electron mobility (open circles), and the corrected values (triangles) after the interfacial conduction effect has been subtracted. The line shows the model fit to the data.

${ }^{1}$ D. C. Look and R. J. Molnar, Appl. Phys. Lett. 70, 3377 (1997).

${ }^{2}$ W. Gotz, J. Walker, L. T. Romano, N. M. Johnson, and R. J. Molnar, Mater. Res. Soc. Symp. Proc. 449, 525 (1997).

${ }^{3}$ D. C. Look, Electrical Characterization of GaAs Materials and Devices (Wiley, New York, 1989).

${ }^{4}$ S. Fung, X. L. Xu, Y. W. Zhao, and M. Gong (unpublished).

${ }^{5}$ S. Fung, X. L. Xu, and Y. W. Zhao, J. Appl. Phys. 84, 2355 (1998).

${ }^{6}$ J. Neugebauer and C. G. van de Walle, Appl. Phys. Lett. 69, 503 (1996).

${ }^{7}$ D. W. Jenkins, J. D. Dow, and M. H. Tsai, J. Appl. Phys. 72, 4130 (1992).

${ }^{8}$ C. Wetzel, T. Suski, J. W. Ager III, E. R. Weber, E. E. Haller, S. Fisher, B. K. Meyer, R. J. Molnar, and P. Perlin, Phys. Rev. Lett. 78, 3925 (1997).

${ }^{9}$ N. F. Mott and W. D. Twose, Adv. Phys. 10, 107 (1961).

${ }^{10}$ T. Matsubara and Y. Toyozawa, Prog. Theor. Phys. 26, 739 (1961).

${ }^{11}$ B. C. Chung and M. Gershenzon, J. Appl. Phys. 72, 651 (1992).

${ }^{12}$ D. M. Hofmann, D. Kovalev, G. Steude, B. K. Meyer, A. Hoffmann, L. Eckey, R. Heitz, T. Detchprom, H. Amano, and I. Akasaki, Phys. Rev. B 52, 16702 (1995).

${ }^{13}$ D. K. Gakill, L. B. Rowland, and K. Doverspike, in Properties of Group III Nitrides EMIS Ser. No. 11, edited by J. H. Edigar (1994), p. 101.

${ }^{14}$ D. C. Look and R. J. Molnar, Appl. Phys. Lett. 70, 3377 (1997).

${ }^{15}$ M. Ilegems and H. C. Montgomery, J. Phys. Chem. Solids 34, 885 (1973).

${ }^{16}$ D. C. Look, J. R. Sizelove, S. Skeller, Y. F. Wu, U. K. Mishra, and S. P. DenBaars, Solid State Commun. 102, 297 (1997). 